\title{
Hepatoprotective Effects of a Chinese Herbal Formula, Longyin Decoction, on Carbon-Tetrachloride-Induced Liver Injury in Chickens
}

\author{
Chunguang Wang, Tie Zhang, Xuemei Cui, Shuang Li, Xinghua Zhao, and Xiuhui Zhong \\ College of Traditional Chinese Veterinary Medicine, Agricultural University of Hebei, Baoding 071001, China
}

Correspondence should be addressed to Xiuhui Zhong; zxh8078@163.com

Received 15 November 2012; Revised 19 January 2013; Accepted 21 January 2013

Academic Editor: Pradeep Visen

Copyright (C) 2013 Chunguang Wang et al. This is an open access article distributed under the Creative Commons Attribution License, which permits unrestricted use, distribution, and reproduction in any medium, provided the original work is properly cited.

\begin{abstract}
The objective of this study is to establish poultry liver injury model induced by $\left(\mathrm{CCl}_{4}\right)$ and seek effective hepatoprotective herbals for clinical application. Different doses of $\mathrm{CCl}_{4}$ dissolved in vegetable oil $(1: 1, \mathrm{~V} / \mathrm{V})$ were injected via pectoral muscle to induce acute liver injury model in chickens. An herbal formula, Longyin decoction, was prepared for hepatoprotection test on chicken acute liver injury models. The pathologic changes of the liver were observed, and the activities of ALT and AST were, respectively, detected to evaluate the hepatoprotective effects of Longyin decoction on chickens. The chicken acute liver injury model was successfully established by injecting $\mathrm{CCl}_{4}$ via pectoral muscle. The best dose of $\mathrm{CCl}_{4}$ inducing chicken liver injury was $4.0 \mathrm{~mL} / \mathrm{kg}$. BW (body weight). The results of qualitative determination by HPTLC showed that the components of Longyin decoction contained Gentian, Capillaries, Gardenia, and Bupleurum root. In the high-dose Longyin group and the middle-dose Longyin group, the pathological changes of the damaged liver were mitigated and the activities of ALT and AST in serum were reduced significantly. Longyin decoction has obvious hepatoprotective effect on acute liver injury induced by $\mathrm{CCl}_{4}$.
\end{abstract}

\section{Introduction}

The liver is the largest substantive gland and an important metabolic organ. It has many important and complex physiological functions, including metabolism, excretion, detoxification, and generating a variety of coagulation factors. With the expanding of intensive farming industry, the disease factor on poultry hazards has become increasingly serious. Poultry liver injury is a clinically common disease. There are many disease causes including nutritional factors, disease factors, parasite factors, and poisoning factors [1]. The main and basic pathologic symptom of many liver diseases is swelling, degeneration, necrosis, and apoptosis of hepatic cells [2]. The poultry liver injury can lead to low performance and even death and has caused a great economic loss in poultry industry. At present, due to the lack of effective hepatoprotective drug in clinic, strengthening the research and development of hepatoprotective drugs has important significance to improve animal health. Chinese herbal medicine has many advantages like little side-effect, low cost, and little drug resistance. Thus, Chinese herbal preparations have been widely used in the animal health and disease prevention and control.

The $\mathrm{CCl}_{4}$ can produce high toxicity in the metabolic process, and thus the vegetable oil solution of $\mathrm{CCl}_{4}$ is commonly used in the establishment of liver injury model [3]. The main acute toxic effects of $\mathrm{CCl}_{4}$ on animal body mainly manifest in inhibiting the central nervous system and damaging the kidney, liver, and lung [4]. The subacute toxicity of $\mathrm{CCl}_{4}$ mainly shows a dose-dependent elevation of the various enzyme levels in serum with the amount of $\mathrm{CCl}_{4}$ [5].

The mice and other mammals are often used as experimental animals to establish liver injury model in the field of human medicine and veterinary medicine [6-8]. With the rapid development of the poultry industry and taking into account the physiological characteristics of poultry, it is of urgent necessity to establish a $\mathrm{CCl}_{4}$-induced liver damage model of the target poultry. This model will reflect 
more accurately the changes of metabolism, function, and histological structures in poultry body.

We established the chicken liver injury model induced by $\mathrm{CCl}_{4}$ to provide basis for practical application of poultry pathology and clinical diagnosis. At the same time we also studied the hepatoprotective effects of Longyin decoction. The best dose of $\mathrm{CCl}_{4}$ and the possible hepatoprotective mechanism of the herbals were investigated in order to provide theoretical basis for research and development of hepatoprotective herbals.

\section{Materials and Methods}

2.1. Chickens. A total of 360 ISA Brown layers at the age of $1 \mathrm{~d}$ were purchased from Shijiazhuang Jinyu Grandfather Chicken Farm. They were fed for $15 \mathrm{~d}$ according to the feeding standard of chicken (NY/T33-2004) promulgated by the Ministry of Agriculture of the People's Republic of China. Then 60 ISA Brown layers at the age of $15 \mathrm{~d}$ were selected to establish liver injury model according to the standards of health and body weight. The other 300 ISA Brown layers were used for hepatoprotecting study of Chinese herbal medicine. All procedures concerning animal treatments and experimentations in this study were reviewed and approved by the Institutional Committee for Ethical Use of Experimental Animals at Hebei Provincial Department of Animal Science.

2.2. Reagents. Longdan Xiegan powder was purchased from the Henan Shangdu Pharmaceutics Co., Ltd. This herbal product was used as a positive control drug, commercially available and documented in the Chinese Pharmacopoeia of Veterinary Drugs.

Reference herbs and reference herbal components were purchased from China Institute of Veterinary Drug Control. Silica gel $\mathrm{G}$ thin plate was purchased from Yantai Chemical Industry Research Institute. Carbon tetrachloride $\left(\mathrm{CCl}_{4}\right)$ was purchased from the Beijing Chemical Reagent Company.

The formula of Longyin decoction includes Capillaries, Gentian, Gardenia, Bupleurum root, and Licorice, where the five herbs are of equivalent weight $(200 \mathrm{~g})$. They were soaked in 10 times weight of distilled water for $30 \mathrm{~min}$ and then brought to boiling. After simmered for $20 \mathrm{~min}$, they were filtered with four layers of gauze and the herbal soup was collected. Five times weight of distilled water was added into the remaining herbals for a second decoction for another $20 \mathrm{~min}$ and then filtered. The herbal soup collected at two times was put together and concentrated on a slow fire until the crude herbal content is $1 \mathrm{~g} / \mathrm{mL}$. Finally, the prepared Longyin decoction was sterilized and stored in refrigerator for use.

\subsection{Establishment of Chicken Acute Liver Injury Model by} Injecting $\mathrm{CCl}_{4}$. A total of 60 healthy chickens were randomly divided into six groups, including five model groups (groups I-V) and blank control group, 10 chickens of each group. On the first and third days, the chickens were, respectively, challenged with the $\mathrm{CCl}_{4}$ peanut oil solution at the volume ratio of $1: 1$. The injection dose of the $\mathrm{CCl}_{4}$ peanut oil solution in groups I, II, III, IV, and V were 1.0, 2.0, 4.0, 8.0, and $16.0 \mathrm{~mL} / \mathrm{kg} \cdot \mathrm{BW}$, respectively. In the blank control group, the chickens were injected with peanut oil $(4.0 \mathrm{~mL} / \mathrm{kg} \cdot \mathrm{BW})$ via pectoral muscle. During the whole trial, the clinical symptoms of these chickens in each group were monitored and recorded. On days 4 and 7, five chickens were selected from each group and the blood samples were collected via wing vein, and then the serum was separated. The activities of the ALT and AST in serum were detected by the automatic biochemical analyzer. The experimental data were analyzed with SPSS 11.5 statistical software. Moreover, the pathological changes of liver and other major organs were observed. The best dose of $\mathrm{CCl}_{4}$ was determined according to the minimum dose of $\mathrm{CCl}_{4}$ which can induce the visible necrotic lesions and can significantly increase serum aminotransferase activity in liver.

2.4. Detection of Longyin Herbal Formula by HPTLC. Four tests for the identification of Gentian, Capillaries, Gardenia, and Bupleurum root was carried out, three sample solutions (including sample solutions 1, 2, and 3) in each test. Preparation of sample solution was as follows: $10.0 \mathrm{~mL}$ Longyin decoction was evaporated to dryness with water bath and the residue was dissolved in $1.0 \mathrm{~mL}$ methanol. Moreover, in the preparation of sample solution for identification of Bupleurum root, the $10.0 \mathrm{~mL}$ Longyin decoction was firstly extracted with n-butanol, and then it was evaporated to dryness and dissolved in $1.0 \mathrm{~mL}$ methanol. Preparation of control drug solution was as follows: each of $1.0 \mathrm{~g}$ reference drug substance (Gentian, Capillaries, or Gardenia) was boiled and filtered; the filtrate was evaporated to dryness and then added $2.0 \mathrm{~mL}$ methanol. The reference drug substance Bupleurum root was firstly extracted with n-butanol, and then it was boiled, filtered and evaporated; finally, it was dissolved in methanol. Each of $1.0 \mathrm{mg}$ gentiopicroside, geniposide, and saikosaponin- $\alpha$ was respectively, dissolved in $1.0 \mathrm{~mL}$ methanol and they were used as control solution. The negative Longyin decoction sample free of the corresponding drug was prepared according to the prescription. The preparation method of negative control solution was the same as that of the above sample solution. Each of $2.0-2.5 \mu \mathrm{L}$ prepared solution was used for identification of Gentian, Capillaries, Gardenia, and Bupleurum root by HPTLC method.

2.5. Protection Test of Longyin Decoction on Liver. A total of 300 chickens were randomly divided into four treatment groups including high-dose Longyin group, middle-dose Longyin group, low-dose Longyin group, and drug control group. The two control groups include model control group and blank control group, 50 chickens of each group. The best dose of $\mathrm{CCl}_{4}$ to induce liver injury was determined according to the liver injury model test. Except the blank control group, the chickens in other groups were injected twice via pectoral muscle with $\mathrm{CCl}_{4}$ peanut oil solution $(4.0 \mathrm{~mL} / \mathrm{kg} \cdot \mathrm{BW})$ on days 1 and 3 . After the last injection (day $3)$, the chickens in each group were treated with different drugs for 5 days consecutively (from days 4 to 8 ). The chickens in the low-dose Longyin group, middle-dose Longyin group, and high-dose Longyin group were treated with different 
doses of the Longyin decoction (1.0, 2.0, and $4.0 \mathrm{~mL} / \mathrm{L}$, resp.) via drinking water. Chickens in the drug control group were treated with Longdan Xiegan powder $(2.5 \mathrm{~g} / \mathrm{kg})$ mixed with feed. Those chickens in the model group and blank control group were not treated with herbals. On the first day after 5 days, herbal treatment (day 9), the blood samples were collected from the chickens in each group via wing vein, and the activities of ALT and AST in serum were detected. After blood sampling, the livers were picked and the lesions were observed. The liver tissues were sliced and stained with HE. The liver histological changes were observed and photographed with the OLYMPUS microscope, and the experimental data were analyzed with SPSS 11.5 statistical software.

\section{Results}

3.1. Chicken Acute Liver Injury Model Induced by $\mathrm{CCl}_{4}$. In the blank control group, the chickens kept good spirit and appetite and did not show any clinical symptoms. After first challenge with $\mathrm{CCl}_{4}$, the chickens in model groups showed slightly decreased reactivity and had no other obvious clinical symptoms. After second challenge, most of the chickens in model groups showed listlessness and decreased feed intake. In groups III, IV, and V, some chickens discharged yellowish green loose stools and showed standing instability and even recumbency. The appetite was recovered on day 6.

Pathological changes of the liver were observed through clinical autopsy. In group I, the liver was slightly larger and showed slightly yellow color (Figure 1). In group II, the superficial necrosis was found in some chicken liver edge (Figure 2). In group III, the multiple needle tip necrosis appeared in chicken liver, the cecal tonsils had scattered bleeding points, and a small amount of bleeding point was observed at injection site and on endocardium or epicardium (Figure 3). In groups IV and V, much more liver lesions were noticed. The liver was significantly larger and harder, and showed large necrotic plaque. The liver edge was blunt thick and khaki. Moreover, the splenomegaly was larger and appeared piebald (Figures 4 and 5). In the blank control group, the liver was dark red, shiny, wet, flexible, and kept normal size (Figure 6).

The activities of ALT and AST in serum were detected. The results showed that the activities of ALT and AST in serum were increased with the increased dose of $\mathrm{CCl}_{4}$. The changes of ALT were earlier and greater than that of AST. After challenge, both ALT activity on day 4 and AST activity on day 7 had extremely significant difference between group III and blank control group $(P<.01)$. These indicated that injecting $\mathrm{CCl}_{4}$ peanut oil solution $(4.0 \mathrm{~mL} / \mathrm{kg} \cdot \mathrm{BW})$ via pectoral muscle could cause increasing of ALT and AST activities in serum.

According to the observation results of clinical symptoms, clinical autopsy, and aminotransferase activity detection, the best dose of $\mathrm{CCl}_{4}$ peanut oil solution inducing chicken acute liver injury was determined and was $4.0 \mathrm{~mL} / \mathrm{kg} \cdot \mathrm{BW}$ (Table 1).

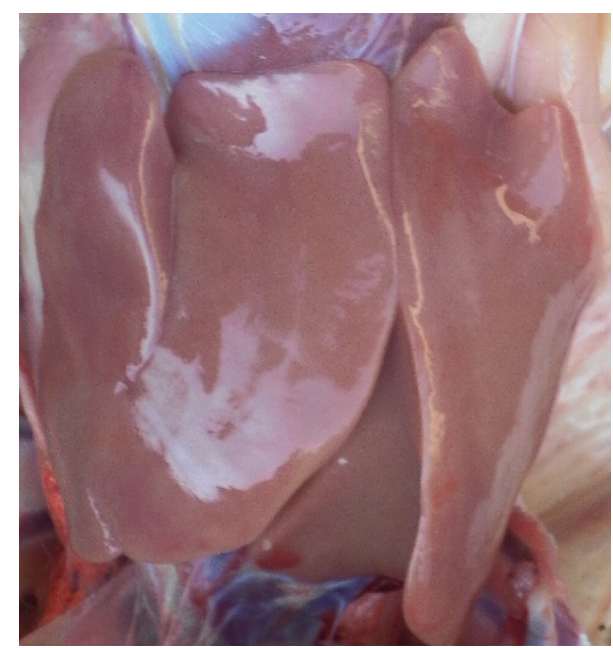

Figure 1: Chicken liver in group I. The liver is slightly larger and yellow in color.

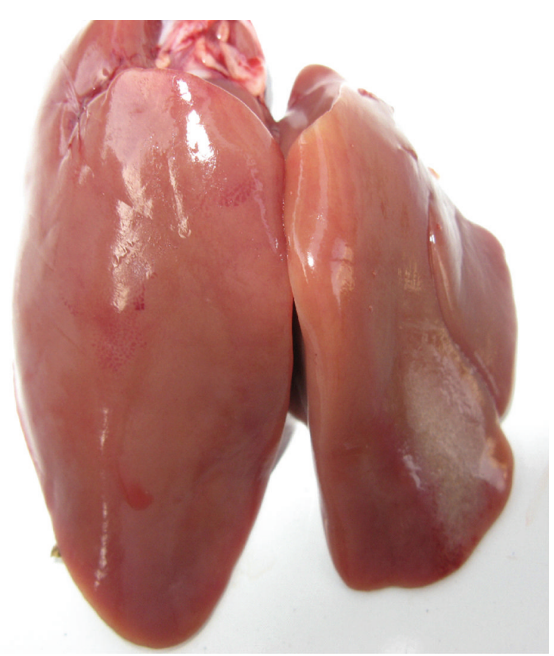

FIGURE 2: Chicken liver in group II. The superficial necrosis is found in liver edge.

3.2. Qualitative Determination of Longyin Decoction. In chromatogram of sample solution, the same color spots were showed in corresponding position with that of control drug solution and control solution. In chromatogram of negative solution, there was no spot found in corresponding position (Figures 7, 8, 9, and 10).

\subsection{Protection Test of Chinese Herbal Medicine on Chicken} Acute Liver Injury. The activities of ALT and AST in each treatment group were lower than that in the model control group with extremely significant differences $(P<.01)$ (Table 2). The activity of ALT in serum had no significant difference between high-dose Longyin group, middle-dose Longyin group, and blank control group $(P>.05)$. The ALT levels in middle-dose Longyin group and low-dose Longyin group were significantly different from that in drug control group $(P<.05)$. In the low-dose Longyin group and 
TABLE 1: Detection of serum biochemical indicators after modeling test.

\begin{tabular}{lcccc}
\hline Groups & \multicolumn{2}{c}{ ALT (U/L) } & \multicolumn{2}{c}{ AST (U/L) } \\
& On day 4 & On day 7 & On day 4 & $217.65 \pm 37.84$ \\
Group I & $2.21 \pm 0.32$ & $2.41 \pm 0.42$ & $207.64 \pm 46.74$ & $233.66 \pm 45.54^{*}$ \\
Group II & $2.30 \pm 0.42$ & $2.55 \pm 0.54^{*}$ & $214.91 \pm 41.09$ & $264.07 \pm 31.29^{* *}$ \\
Group III & $3.22 \pm 0.35^{* *}$ & $3.75 \pm 0.40^{* *}$ & $232.41 \pm 32.46$ & $284.64 \pm 35.06^{* *}$ \\
Group IV & $3.54 \pm 0.40^{* *}$ & $4.22 \pm 0.33^{* *}$ & $248.08 \pm 53.62^{*}$ & $298.07 \pm 42.70^{* *}$ \\
Group V & $3.88 \pm 0.34^{* *}$ & $4.83 \pm 0.57^{* *}$ & $265.22 \pm 39.74^{* *}$ & $194.39 \pm 25.16$ \\
Bland control group & $2.11 \pm 0.25$ & $2.12 \pm 0.35$ & $198.55 \pm 33.62$ & \\
\hline
\end{tabular}

Values are mean $\pm \mathrm{SE}, n=5$.

${ }^{*}$ means significant difference $(P<0.05) ;{ }^{* *}$ Means extremely significant difference $(P<0.01)$.

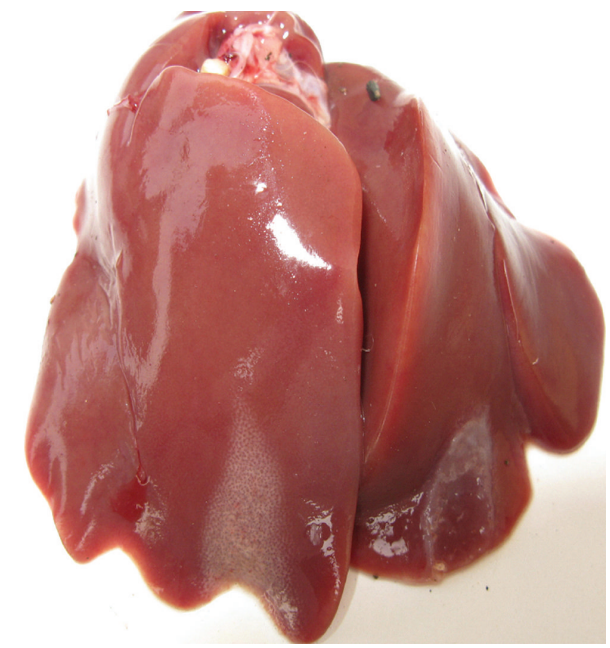

FIGURE 3: Chicken liver in group III. The multiple needle tip necrosis appears in liver.

TABLE 2: Detection of serum biochemical indicators after treatment by Longyin decoction.

\begin{tabular}{lcc}
\hline Groups & ALT (U/L) & AST (U/L) \\
\hline Low-dose Longyin group & $2.67 \pm 0.53^{\mathrm{BCb}}$ & $239.48 \pm 47.96^{\mathrm{Bb}}$ \\
Middle-dose Longyin group & $2.42 \pm 0.59^{\mathrm{BCDc}}$ & $221.24 \pm 50.62^{\mathrm{BCcd}}$ \\
High-dose Longyin group & $2.37 \pm 0.67^{\mathrm{CDc}}$ & $216.76 \pm 49.01^{\mathrm{Cd}}$ \\
Drug control group & $2.69 \pm 0.76^{\mathrm{Bb}}$ & $237.81 \pm 38.32^{\mathrm{BCbc}}$ \\
Model control group & $3.83 \pm 0.46^{\mathrm{Aa}}$ & $273.50 \pm 36.65^{\mathrm{Aa}}$ \\
Blank control group & $2.19 \pm 0.58^{\mathrm{Dc}}$ & $192.40 \pm 30.92^{\mathrm{De}}$ \\
\hline
\end{tabular}

Values are mean \pm SE, $n=50$.

Different lowercase letters mean significant difference $(P<0.05)$; different majuscule letters mean extremely significant difference $(P<0.01)$.

drug control group, the ALT levels have extremely significant differences from that in blank control group $(P<.01)$.

The activity of AST in serum in each treatment group was significantly different from that in blank control group $(P<.01)$. There was no significant difference of AST levels between high-dose Longyin group and middle-dose Longyin group $(P>.05)$. The AST level of middle-dose Longyin group was significantly different from that of low-dose Longyin group $(P<.05)$.

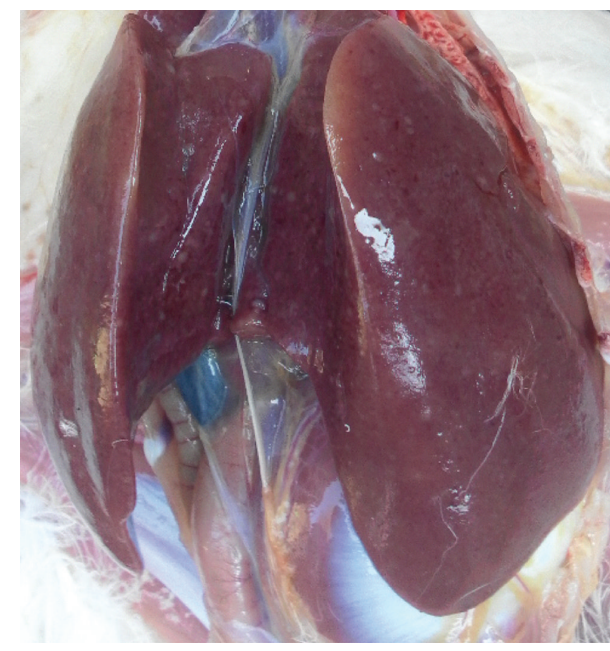

FIgURE 4: Chicken liver in group IV. The liver is larger and harder and shows large necrotic plaque.

At necropsy the color and volume of liver in blank control group were normal. In the model control group, the liver showed multiple needle tip necrosis. In the high-dose Longyin group, a few chickens showed slightly yellow at edge of liver. In the middle-dose Longyin group, low-dose Longyin group, and the drug control group, a small amount of needle tip necrosis appeared in chicken livers.

The liver histopathology of chickens in each group was examined. In the blank control group, the hepatic lobules centered around the central vein, the hepatocyte tubes being surrounded by liver cells, showed radially walk to the lobule edge. There was a small amount of red blood cells and white blood cells in the liver sinusoids between the hepatocyte tubes. The liver cells were cube with plump cytoplasm and normal structure, and the nucleus was in regular shape and located in the center of the cell (Figure 11).

In the model control group, the hepatocyte tubes were arranged haphazardly with severe congestion and inflammatory cell infiltration. Liver cells had irregular contour with many vacuoles formed by fatty degeneration. Some areas of liver showed necrosis, cell rupture, nuclear condensation, fragmentation, and aggregation (Figure 12).

In the high-dose Longyin group, the hepatic lobules had clear structure, the hepatocyte tubes were arranged 


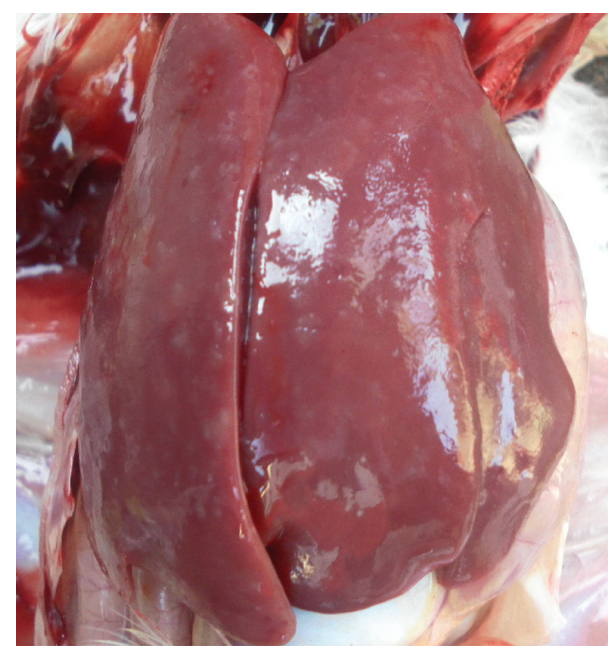

FIgure 5: Chicken liver in group V. The liver is larger and harder and shows large necrotic plaque.

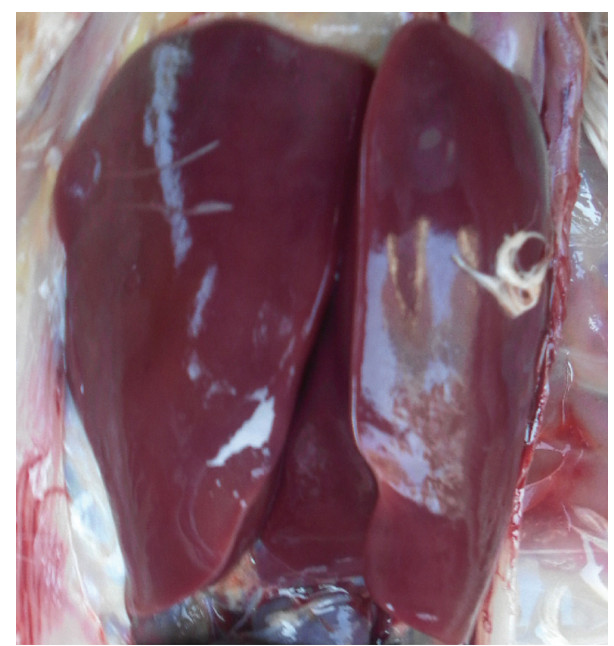

FIgURE 6: Chicken liver in blank control group. The liver is red, shiny, wet, flexible, and keeping normal volume.

orderly and closely, and the liver sinusoids showed the mild congestion (Figure 13). In the middle-dose Longyin group, the hepatic lobule structure was normal, the hepatocyte tubes arranged orderly but slightly loose with mild congestion, and the cytoplasm was reduced (Figure 14). In the low-dose Longyin group, the the hepatocyte tubes were arranged in disorder with congestion and inflammatory cell infiltration, and the liver cells showed pale watery cytoplasm (Figure 15).

In the drug control group, the grade of liver lesions was close to those in the low-dose Longyin group, we also found the congestion and inflammatory cell infiltration, as well as granular degeneration of the liver cells (Figure 16).

\section{Discussion}

4.1. Damage Effects of $\mathrm{CCl}_{4}$ on Liver. $\mathrm{CCl}_{4}$ is a strong proliver toxicant and can produce lively trichloromethyl radicals and

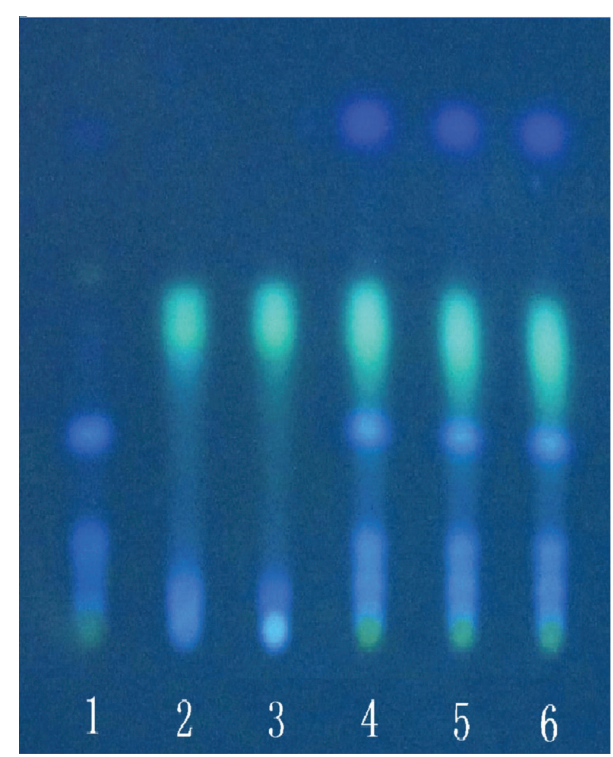

FIGURE 7: Thin-layer chromatogram of Gentian in Longyin decoction. 1: negative control solution free Gnetian; 2: control solution of gentiopicroside; 3: reference drug substance of Gentian; 4-6: sample solutions 1,2 , and 3 .

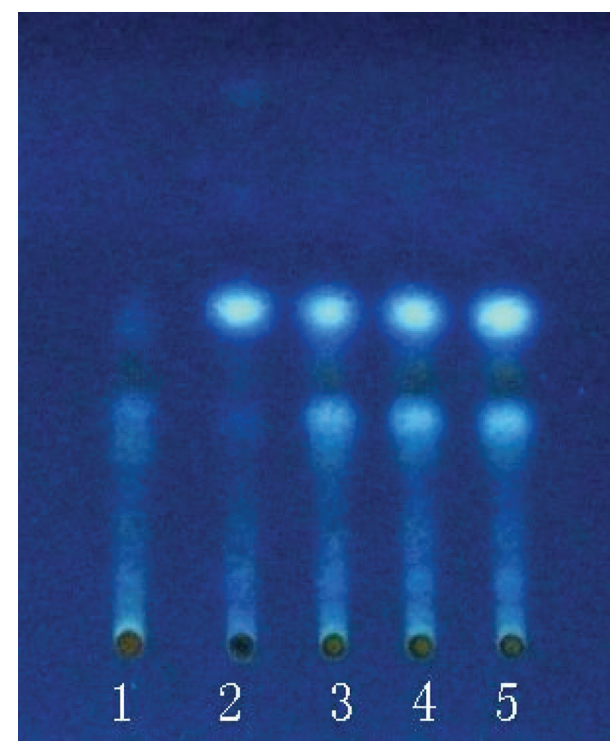

FIGURE 8: Thin-layer chromatogram of Capillaries in Longyin decoction. 1: negative control solution free Capillaries; 2: reference drug substance of Capillaries; 3-5: sample solution 1, 2, and 3.

chlorine-free radicals after getting into body via different pathways. It can cause the lipid peroxidation of endoplasmic reticulum membrane and cell membrane, and then damage the liver cell. In addition, the $\mathrm{CCl}_{4}$ can reduce the degeneration, necrosis, fibrosis, and canceration of liver [9]. Therefore, we can observe the inflammatory cell infiltration, fatty degeneration, cloudy swelling, ballooning degeneration, and liver necrosis in liver slices. The destruction of liver cell membrane structure and functional integrity causes the increase of the soluble enzymes (ALT and AST) activity. Then 


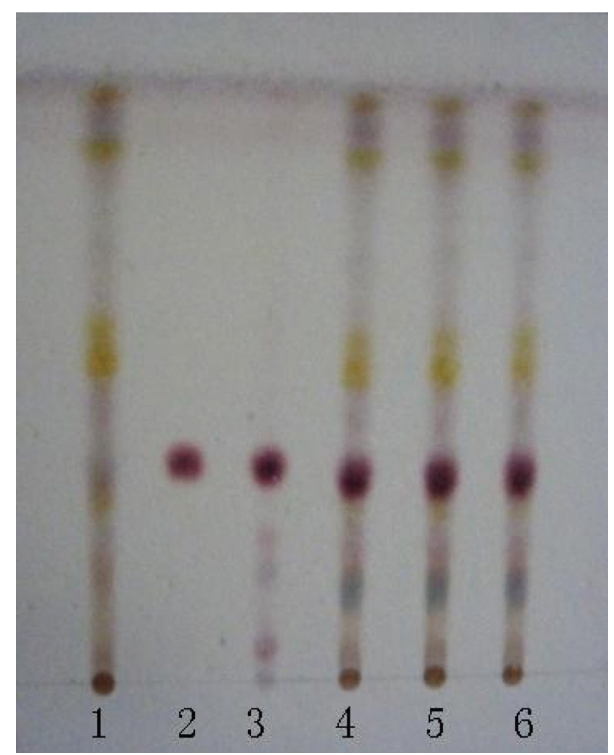

FIGURE 9: Thin-layer chromatogram of Gardenia in Longyin decoction. 1: negative control solution free Gardenia; 2: Control solution of geniposide; 3: reference drug substance of Gardenia; 4-6: sample solution 1,2 , and 3 .

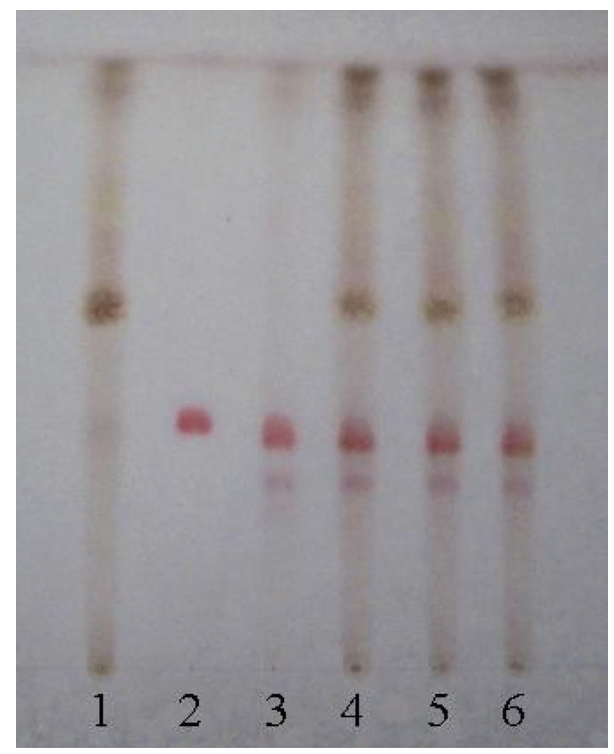

FIGURE 10: Thin-layer chromatogram of Bupleurum root in Longyin decoction. 1: negative control solution free Bupleurum root; 2: control solution of saikosaponin- $\alpha$; 3: reference drug substance of Bupleurum root; 4-6: sample solution 1, 2, and 3.

these soluble enzymes get into blood from the liver cells thus, causing the increase of serum enzyme activity. Therefore, the activities of ALT and AST in serum are a sensitive index of liver damage and can reflect the degree of liver damage and necrosis to a certain extent [10].

In this study, injecting $\mathrm{CCl}_{4}$ oil solution $(>4.0 \mathrm{~mL} / \mathrm{kg} \cdot \mathrm{BW})$ via pectoral muscle can result in chicken liver substantial necrotic lesions and great increases of ALT and AST levels

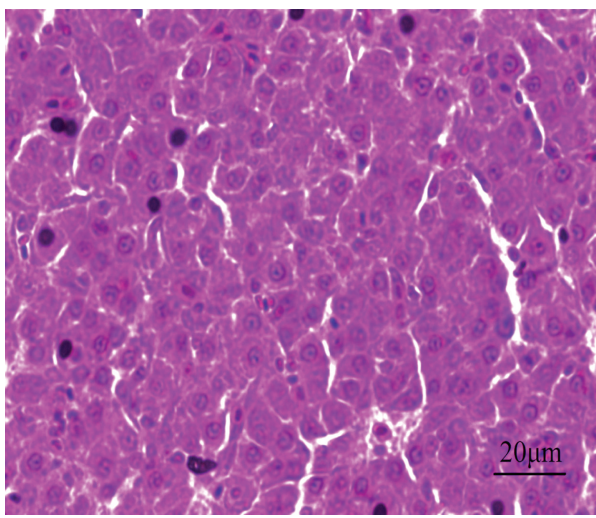

FIGURE 11: HE staining of chicken liver slice in blank control group. The hepatic lobule has complete structure and the liver cell has regular shape, $400 \mathrm{x}$.

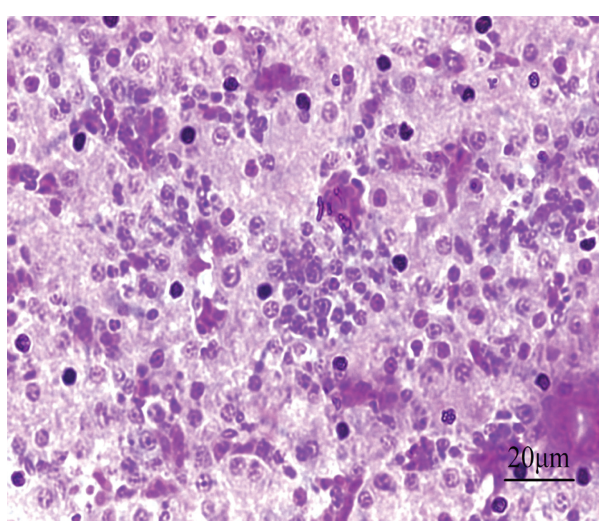

FIGURE 12: HE staining of chicken liver slice in model control group. The hepatocyte tubes arranged haphazardly with severe congestion and inflammatory cell infiltration, liver cells appear necrosis with nuclear fragmentation, 400x.

in serum. In early period of trial, the change speed of ALT is greater than that of AST. However, we had verified that the damage of $\mathrm{CCl}_{4}$ on chicken liver shows a toxic cumulative effect, namely, the chicken liver injury and increase of serum transaminase levels induced by $\mathrm{CCl}_{4}$ require certain duration of action and a higher challenge dose. It indicates that chicken has certain tolerability to $\mathrm{CCl}_{4}$ oil solution.

4.2. Protection Effects of Longyin decoction on Liver. According to the concept of traditional Chinese Veterinary medicine, liver damage is of $Q i$ stagnation pattern. The pathogenesis is damp-heat stagnation resulting in liver $Q i$ difficulties in moving, and the treatment principle should be to dispel damp-heat and smooth the liver [11]. A large number of clinical practices and experimental researches have confirmed that the Chinese herbal medicine has significant protective effects on liver injury and has unique advantages on removing jaundice and liver protection therapy. The treatment of Chinese herbal medicine on liver injury is mainly manifested in the inhibition of lipid peroxidation, stabilization of biological membrane, improving hepatic 


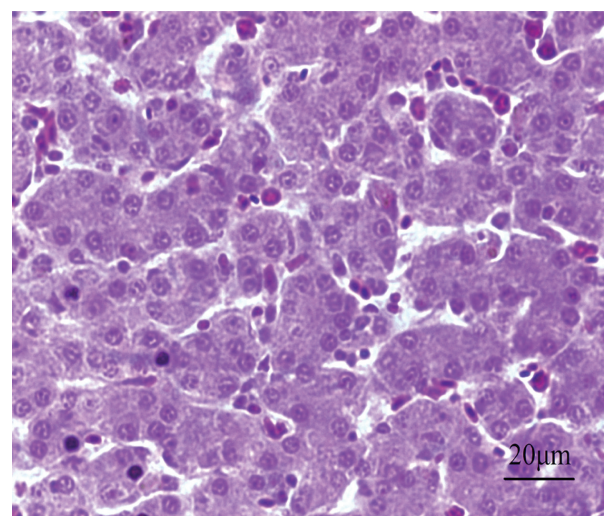

FIGURE 13: HE staining of chicken liver slice in high-dose Longyin group. The hepatic lobules have clear structure, the hepatocyte tubes arranged orderly and closely, 400x.

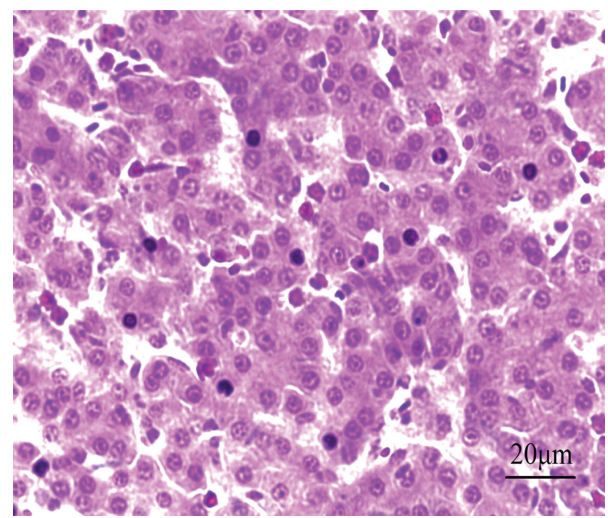

FIGURE 14: HE staining of chicken liver slice in middle-dose Longyin group. The hepatocyte tubes arranged orderly but slightly loose with mild congestion, the cytoplasm is reduced, 400x.

microcirculation, inhibiting liver cell apoptosis, and inflammatory cytokines [12]. Previous studies have reported many single Chinese herbs with hepatoprotective effects as well as their active ingredients, including Scutellaria, Astragalus, Herba Rhodiola, Polygonum cuspidatum, Radix lithospermi, Angelica sinensis, Radix salviae miltiorrhizae, Cordyceps polysaccharide, Polysaccharide of Grifola Frondosa (PGF), Geranium flavonoids, Rhizoma Anemarrhenae total flavone, Momordica glycoside, tetramethylpyrazine (TMP), Fly thistle element, schizandrin, tetrandrine glycyrrhizin, and others $[1,13-20]$. Luo et al. have verified that the skullcap and baicalin have protective effects on rat primary hepatocytes damaged by ethanol. Particularly, the optimal concentration in vitro of the baicalin is $0.05 \mu \mathrm{g} / \mathrm{mL}$ [13]. The $P G F$ can inhibit the liver cell apoptosis by regulating expression of apoptosisrelated protein $\mathrm{Bcl}-2$ and $\mathrm{Bax}$. It also can reduce the activity of serum transaminase, increase the SOD content and decrease the MDA content to enhance the ability of scavenging free radicals [1].

In addition, a great number of studies have demonstrated that the protective effect of compound Chinese herbal medicine on liver is more comprehensive. The commonly

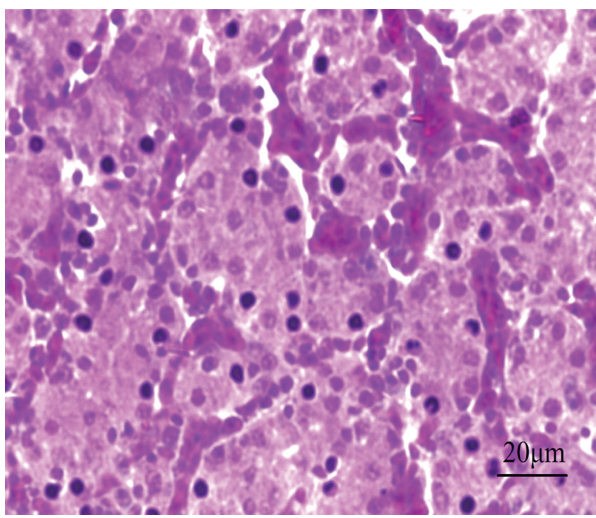

FIGURE 15: HE staining of chicken liver slice in low-dose Longyin group. The hepatocyte tubes arranged in disorder with congestion and inflammatory cell infiltration, and the liver cells show pale watery cytoplasm, 400x.

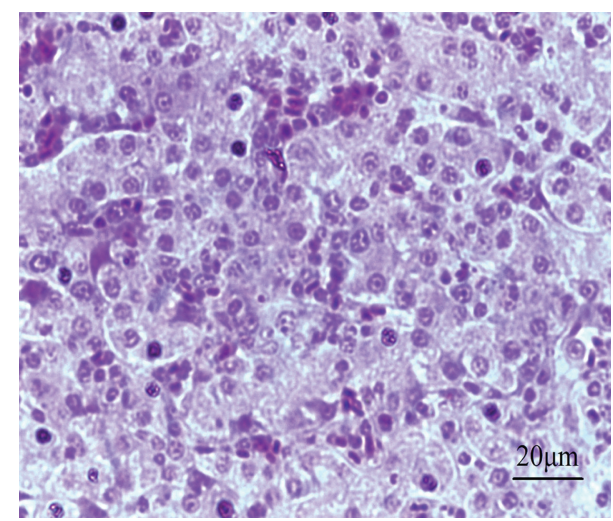

FIGURE 16: HE staining of chicken liver slice in drug control group. The liver shows congestion and inflammatory cell infiltration, and the liver cell shows granular degeneration, 400x.

used Chinese herbal prescription includes the modified Xiaochaihu Decoction, Longdan Xiegan powder, compound Caiyu decoction, Peony and Licorice decoction, Yinchenhao decoction, Glycyrrhizae decoction for purging stomach fire, and Sini decoction [21-27].

The Longdan Xiegan decoction can decrease the serum lactate dehydrogenase (LDH) and ALT levels of mice with liver injury and reduce the cell degeneration and liver necrosis induced by $\mathrm{CCl}_{4}[22]$. The compound Caiyu decoction can effectively prevent the immunologic damage of mouse liver induced by BCG + lipopolysaccharide (LPS) and significantly decrease the AST and ALT levels in serum [23].

Many previous researches on hepatoprotective effect of traditional Chinese medicine focus on mammalian (mouse and rat), and research subjects which focuses on poultry has not been reported.

The Longyin decoction was modified and prepared according to the classic prescription of traditional Chinese medicine "Oriental wormwood Decoction" in Waitai miyao written by Wang Tao of Tang Dynasty. And it includes Capillaries, Gentian, Gardenia, Bupleurum root, and Licorice [28]. 
Gentian which refers to the root and rhizome of Gentiana manshurica Kitag.; Gentiana scabra Bge.; Gentiana triflora Pall., or Gentiana rigescens Franch. ex Hemsl. Capillaries was whole herb or aboveground parts of Artemisia scoparia Waldst. et Kit. or Artemisia capillaris Thumb. Gardenia is the dried ripe fruit of Gardenia jasminoides Elli. Bupleurum root is the dried root of Bupleurum chinense DC. or Bupleurum scorzonerifolium Willd. Licorice refers to the dried root and rhizome of Glycyrrhiza uralensis Fisch.; Glycyrrhiza inflate Bat. or Glycyrrhiza glabra L. Long dan (Gentian), and Yin chen (Capillaries) functions to treat jaundice according to the Chinese herbal book, while Zhi zi (Gardenia), chai hu (Bupleurum root), and gancao (Licorice) work together to assist Long dan and Yin chen. Take the first words of the two main herbs, hence the formula name is Longyin decoction. In the hepatoprotective test of Longyin decoction on liver, the serum transaminase activity and the liver microstructure in each experimental group were detected and observed, respectively. The results showed that the Longyin oral liquid could reduce liver necrosis and decrease serum transaminase activity (ALT and AST) and had protective effects on liver injury induced by $\mathrm{CCl}_{4}$.

\section{Acknowledgments}

This study was financially supported by the Ministry of Science and Technology, China (project no. 2011BAD34B02) and Hebei Key Technology R\&D Program Grant (project no. 10220414) from Department of Science \& Technology, Hebei Province, China.

\section{References}

[1] Y. Z. Wang, The protective effect of polysaccharide of grifola frondosa on carbon tetrachloride induced liver injury and its mechanism [M.S. thesis], Shandong University, 2010.

[2] C. S. Xue, Q. H. Yang, and S. J. Liu, "Protective effect of Jiangzhi Ninggan capsule on acute hepatic injury induced by Dgalactosamine in mice," Lishizhen Medicine and Materia Medica Research, vol. 20, pp. 295-296, 2009.

[3] X. Y. Wang, G. L. Hu, and C. Y. Zhang, "Protective effect of Baogan Hupi liquid on acute liver injury induced by carbon tetrachloride," Chinese Journal of Traditional Veterinary Science, vol. 2, pp. 10-11, 2006.

[4] R. Shulze and H. Kappus, "Lyses of erythrocytes as a result of microtonal lipid Peroxidation induced by $\mathrm{CCl}_{4}$ or $\mathrm{FeCl}_{2}$," Research Communications in Chemical Pathology and Pharmacology, vol. 27, pp. 129-137, 1980.

[5] J. R. Hayes, L. W. Condie, and J. F. Borzelleca, "Acute, 14day repeated dosing, and 90-day subchronic toxicity studies of carbon tetrachloride in CD-1 mice," Fundamental and Applied Toxicology, vol. 7, no. 3, pp. 454-463, 1986.

[6] A. Mondal, S. K. Karan, T. Singha, D. Rajalingam, and T. K. Maity, "Evaluation of hepatoprotective effect of leaves of Cassia sophera linn," Evidence-Based Complementary and Alternative Medicine, vol. 2012, Article ID 436139, 5 pages, 2012.

[7] L. Xu, J. Gao, Y. Wang et al., "Myrica rubra extracts protect the liver from $\mathrm{CCl}_{4}$-induced damage," Evidence-Based Complementary and Alternative Medicine, vol. 2011, Article ID 518302, 8 pages, 2011.
[8] X. Zhao, H. Jia, S. Yang et al., "Salvianolic acid B reducing portal hypertension depends onmacrophages in isolated portal perfused rat livers with chronic hepatitis," Evidence-Based Complementary and Alternative Medicine, vol. 2012, Article ID 786365, 8 pages, 2012.

[9] Z. G. Wang, Studies on extracting technology of TFG and biological activity of Geranium wilfordii maxim [M.S. thesis], Yangzhou University, 1986.

[10] J. Liu, D. J. Liu, F. F. Qiao, J. N. Ni, Y. J. Lv, and E. D. Bao, "Establishment of experimental animal model of chicken liver and kidney damages induced by carbon tetrachloride," Journal of Nanjing Agricultural University, vol. 31, pp. 117-120, 2008.

[11] D. Liu, H. F. Tang, S. Q. Zhang, Y. Di, J. Meng, and J. W. Wang, "Protective effect of concentrated tablet of Polygonum cuspidatum on liver injury in mice," Lishizhen Medicine and Materia Medica Research, vol. 18, pp. 3034-3035, 2007.

[12] R. F. Yang, Establishment of mice liver injury model induced by $D M N$ and protective effect of Chinese medicine on liver [Doctor thesis], Guangzhou University of Chinese Medicine, 2008.

[13] D. S. Luo, H. H. Zheng, and Q. Liu, "Experimental research of Huangqin elixation's protective effect on liver injury induced by carbon tetrachloride in rats," Journal of Xianning Medical College, vol. 15, p. 92, 2001.

[14] J. T. Lu, Y. Yang, W. Wei, and Z. M. Chen, "Protective effects of compound astragalus extract on chemical and immunological liver injury in mice," Chinese Journal of Information on Traditional Chinese Medicine, vol. 1, pp. 32-34, 2008.

[15] H. Z. Lv, S. F. Huang, J. M. Cui, G. Li, and G. C. Piao, "Protective effect of ultra fine powder of Rhodiola sacra on acute injury in mice," Journal of Medical Science Yanbian University, vol. 30, pp. 259-261, 2007.

[16] Z.-D. Liu, X.-G. Zhao, and S.-L. Yiu, "Study progress in treatment of hepatic injury and prospect," Guiding Journal of TCM, vol. 10, pp. 81-87, 2007.

[17] Y.-P. Shang, S.-Y. Fang, J.-F. Ge, L. Zhang, and J. Li, "Protective action of cordyceps polysaccharides on immunological liver injury in mice," West China Journal of Pharmaceutical Sciences, vol. 22, pp. 654-655, 2007.

[18] M. M. Li, T. Jiang, J. C. Hang, X. S. Yao, and Y. B. Li, "Protective effect of Rhizoma anemarrhenae total flavone on liver injury in mice induced by acetic acid," Chinese Traditional and Herbal Drugs, vol. 39, pp. 252-255, 2008.

[19] Q. Wang and D. Xiao, "Protective effect of Mogrosides on chronic liver injury in rats," Guangxi Journal of Traditional Chinese Medicine, vol. 30, pp. 54-56, 2007.

[20] S. M. Zhu and Z. P. Tang, "Research progress of protective effect of Yinchenhao decoction on liver," Shanghai Journal of Traditional Chinese Medicine, vol. 42, pp. 73-74, 2008.

[21] B. Xie, D. J. Yu, and S. M. Cheng, "Protective effect of modified minor Radix bupleuri decoction on liver injury induced by paracetamol," Lishizhen Medicine and Materia Medica Research, vol. 19, pp. 130-131, 2008.

[22] M. Kouadir, Study of hepatoprotective and anti-inflammatory effects of Long Dan Xie Gan decoction and their mechanisms [M.S. thesis], Yangzhou University, 2007.

[23] H.-X. Wang, Z.-Q. Li, and F.-X. Lu, "Effect of compound Caiyu decoction on immune system of mice with immunological liver injury," Chinese Journal of Integrated Traditional and Western Medicine on Liver Disease, vol. 17, pp. 288-289, 2007.

[24] L. Di, X.-Y. Liu, H.-F. Chang, Y.-S. Wang, and Q.-G. Zhang, "Protective effect of Peony and Licorice decoction on acute liver 
injury in mice," Pharmacology and Clinics of Chinese Materia Medica, vol. 23, pp. 4-5, 2007.

[25] X.-Q. Zhu and S. S. Lei, "Protective effect of teramethylpyrazineon chemical liver injury in mice," Liaoning Journal of Traditional Chinese Medicine, vol. 35, pp. 138-140, 2008.

[26] J.-N. Zhao, C.-M. Gong, and Y.-J. Song, "Protective effect of licorice Xiexin decoction on experimental liver injury," Pharmacology and Clinics of Chinese Materia Medica, vol. 14, p. 13, 1998.

[27] C.-X. Zhou, Q. Xu, and J. S. Cao, "Study on mechanism of improving cell immunological liver injury of Sinisan," Chinese Journal of Basic Medicine in Traditional Chinese Medicine, vol. 8, p. 47, 2002.

[28] S. Wang, Eleven Herbal Formulas for Joudice, vol. 14 of Waitai Miyao, Peaple’s Medical, Beijing, China, 1955. 


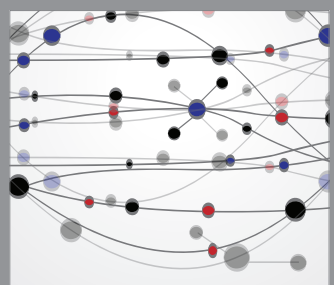

The Scientific World Journal
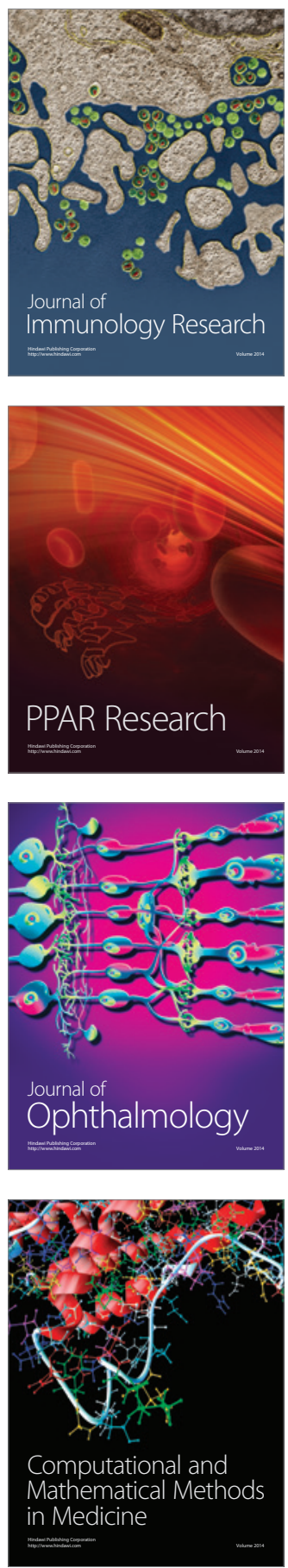

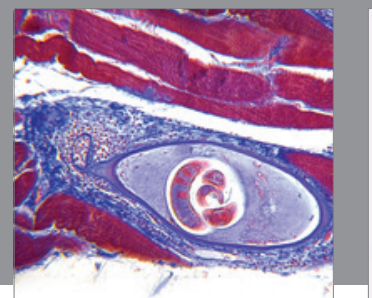

Gastroenterology

Research and Practice
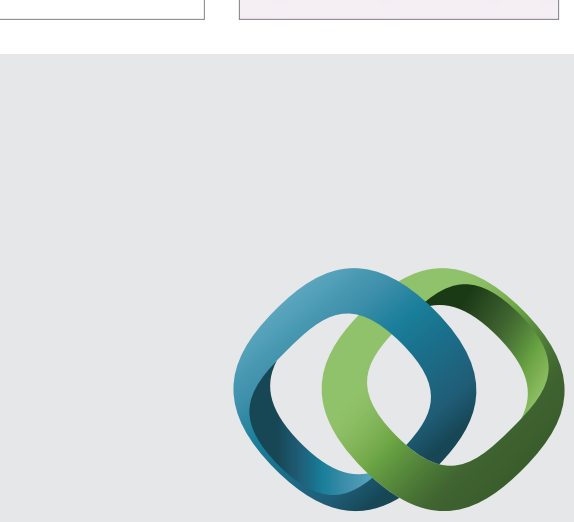

\section{Hindawi}

Submit your manuscripts at

http://www.hindawi.com
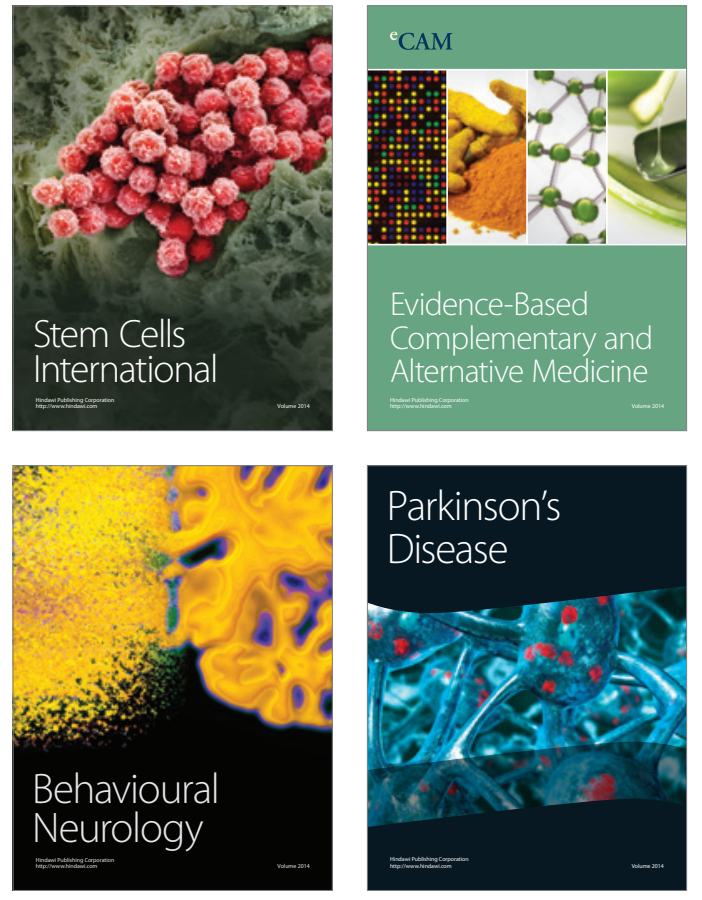
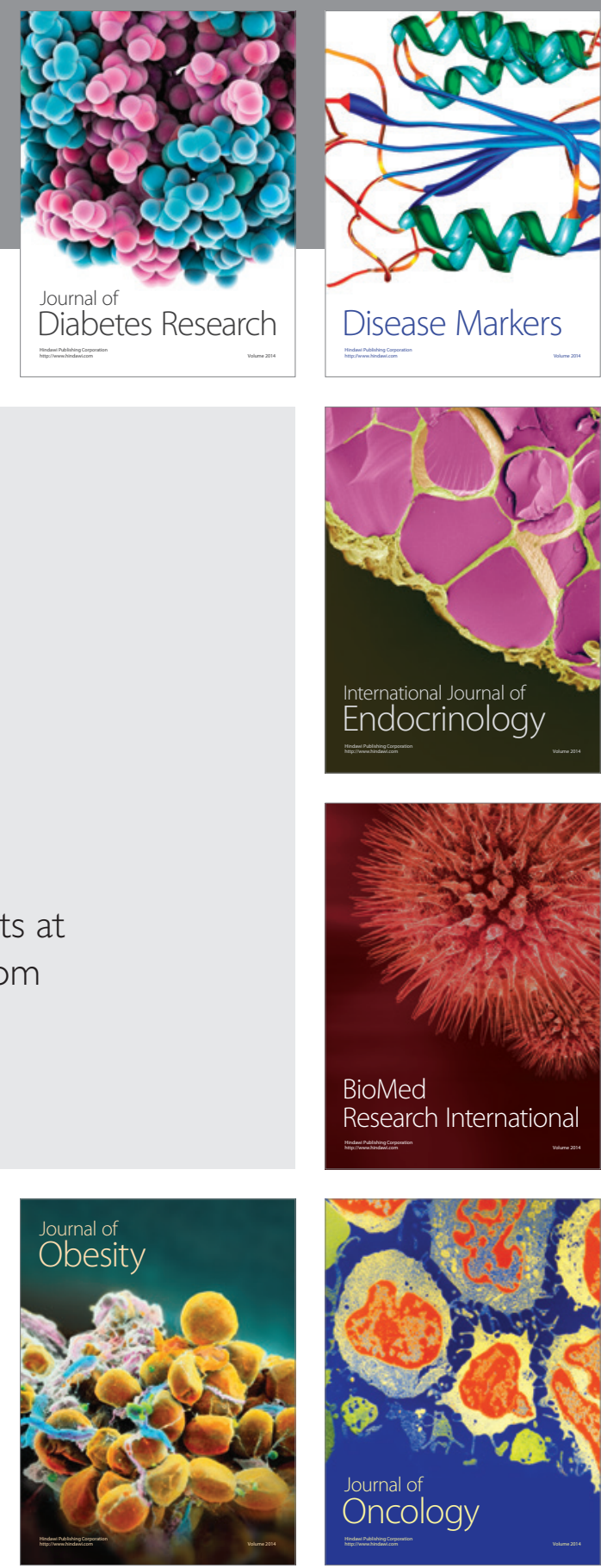

Disease Markers
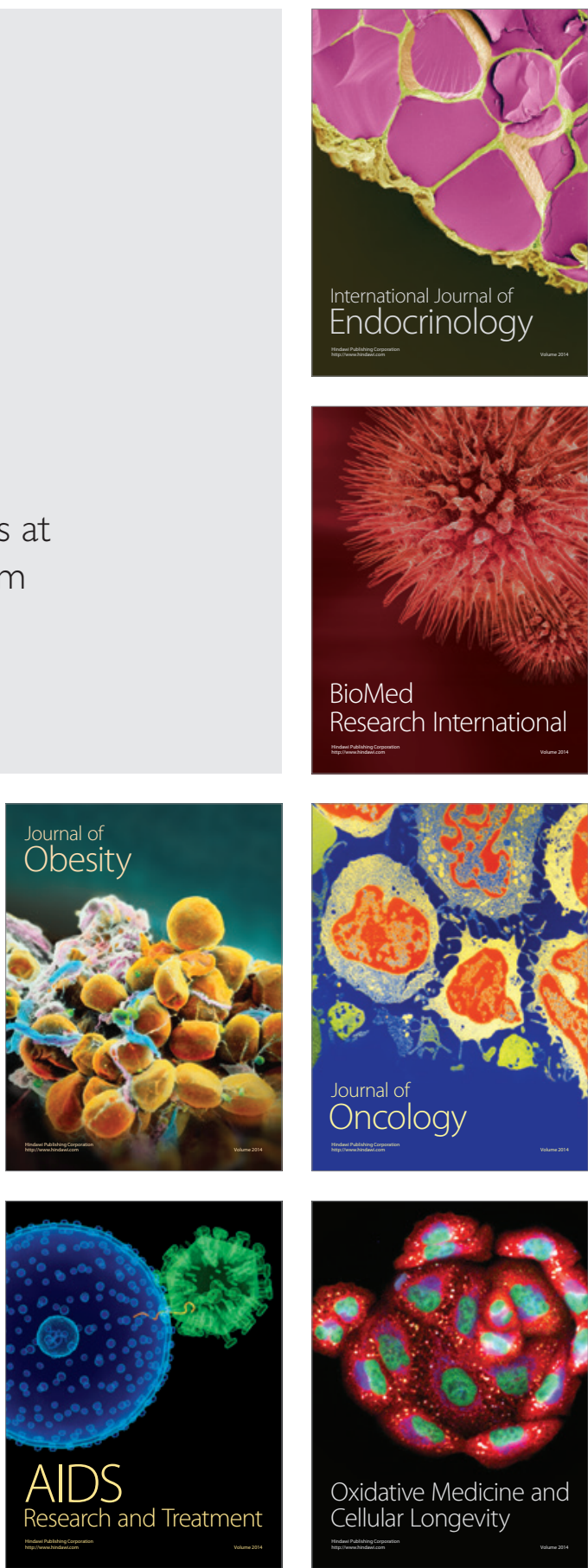